\title{
Alimentos Orgânicos: Qualidade Nutritiva e Segurança do Alimento
}

\author{
Renata Galhardo Borguini ${ }^{1}$, Elizabeth A. Ferraz da Silva Torres ${ }^{2}$
}

‘Orgânico' é um termo de rotulagem que indica que o alimento é produzido de acordo com normas específicas que vetam o uso de quaisquer agroquímicos e que está certificado por uma agência devidamente constituída. Esta revisão discute as distinções entre os alimentos orgânicos e convencionais, com respeito à qualidade nutritiva e à segurança do alimento, e evidencia a existência de diversas diferenças qualitativas.

Palavras-chave: alimentos orgânicos; qualidade nutritiva; resíduos de pesticidas.

\section{Organic Food: Nutritional Quality and Food Safety}

'Organic food' is a labeling term indicating that the food has been produced in accordance with specific norms that exclude the use of any agrochemicals and is certificated by an appropriate agency. This review discusses the distinctions between organic foods and conventional foods with respect to the nutritive quality and food safety and asserts that several qualitative differences do exist.

Keywords: organic food; nutritional quality; pesticide residues.

${ }^{1}$ Agência Paulista de Tecnologia dos Agronegócios. Secretaria de Agricultura e Abastecimento do Estado de São Paulo.

${ }^{2}$ Departamento de Nutrição. Faculdade de Saúde Pública (USP) 


\section{Introdução}

Têm sido observados sinais que evidenciam uma mudança de hábito alimentar entre os brasileiros, na direção de uma maior demanda por produtos orgânicos. A julgar pela presença dos orgânicos nas gôndolas de supermercados, estima-se que exista um potencial de mercado de expressiva magnitude para estes produtos. Tais observações, por si mesmas, chamam a atenção para o potencial deste novo nicho de consumo e para a necessidade da implementação de análises sobre o tema [1].

Há um mercado potencial para os produtos orgânicos, uma vez que existe resistência de uma parcela da população em manter a aquisição e consumo de alguns alimentos convencionais, como tomate, morango e batata, cujo cultivo reconhecidamente envolve o emprego de substanciais quantidades de adubos sintéticos e pesticidas [2]. No entanto, existem controvérsias sobre os alimentos orgânicos, principalmente, quando são classificados como mais nutritivos e seguros [3], devido à escassez de dados científicos que assegurem tais vantagens em relação ao convencional.

Orgânico é um termo de rotulagem que indica que os produtos são produzidos atendendo às normas da produção orgânica e que estão certificados por uma estrutura ou autoridade de certificação devidamente constituída. A agricultura orgânica se baseia no emprego mínimo de insumos externos. No entanto, devido à contaminação ambiental generalizada, as práticas de agricultura orgânica não podem garantir a ausência total de resíduos. Contudo, é possível aplicar métodos que visem à redução, ao mínimo, da contaminação do ar, do solo e da água[4].

Considerando-se o aumento da demanda e também do interesse do consumidor pelos produtos da agricultura orgânica, esta revisão visa abordar aspectos relacionados à qualidade nutritiva e à segurança dos alimentos orgânicos.

\section{Alimentos orgânicos: legislação, certificação e mercado}

Segundo Souza [5], a busca por alimentos provenientes de sistemas de produção mais sustentáveis, como os métodos orgânicos de produção, é uma tendência que vem se fortalecendo e se consolidando mundialmente.

No Brasil, o sistema orgânico de produção está regulamentado pela Lei Federal no 10.831, de 23 de dezembro de 2003, que contém normas disciplinares para a produção, tipificação, processamento, envase, distribuição, identificação e certificação da qualidade dos produtos orgânicos, sejam de origem animal ou vegetal. De acordo com a referida Lei, considera-se sistema orgânico de produção agropecuária todo aquele em que são adotadas técnicas específicas, mediante a otimização do uso dos recursos naturais e socioeconômicos disponíveis e o respeito à integridade cultural das comunidades rurais, tendo por objetivo a sustentabilidade ecológica e econômica, a maximização dos benefícios sociais, a minimização da dependência de energia nãorenovável, empregando, sempre que possível, métodos culturais, biológicos e mecânicos, em contraposição ao uso de materiais sintéticos, a eliminação do uso de organismos geneticamente modificados e radiações ionizantes, em qualquer fase do processo de produção, processamento, armazenamento, distribuição e comercialização, e a proteção do meio ambiente [6].

De acordo com Torjusen et al. [7], a agricultura orgânica tem sido praticada desde a década de 20, inicialmente como uma resposta ao processo de industrialização da agricultura, marcado pela tecnificação. Com relação às metas da agricultura orgânica, as mais relevantes para os consumidores são: a não utilização de pesticidas e fertilizantes químicos sintéticos, de organismos geneticamente modificados, de estimulantes de crescimento sintéticos e de antibióticos, além do uso restrito de aditivos em alimentos processados.

De acordo com Souza [5], quanto menos direta for a relação entre produtores e consumidores, maior será a necessidade de instrumentos formais de certificação da produção. Isso ocorre devido à maior distância entre os agentes e à dificuldade para a comprovação das características inerentes a esses produtos.

Existem, mundialmente, centenas de agências 
de certificação orgânica, as quais estabelecem seus próprios padrões de produção e processo de certificação. No Brasil, existem cerca de 15 certificadoras. Um pequeno número destas agências obteve autorização da International Federation of Organic Agricultural Movements (IFOAM), baseado na constatação de que operavam em concordância com os padrões básicos da IFOAM.

Os produtos comercializados in natura, sobretudo as hortaliças, são os mais expressivos na produção orgânica nacional [8]. Entre os produtos orgânicos destinados à exportação, merecem destaque a soja, café, cacau, açúcar mascavo, ervamate, suco de laranja, mel, frutas secas, castanha de caju, óleos essenciais, óleo de palma, frutas tropicais, palmito, guaraná e arroz.

Estima-se que $90 \%$ dos agricultores orgânicos no país sejam classificados como pequenos produtores ligados a associações e grupos de movimentos sociais. Os $10 \%$ restantes são representados pelos grandes produtores vinculados a empresas privadas. Os agricultores familiares são responsáveis por $70 \%$ da produção orgânica, com maior expressão na região sul do país, enquanto na região sudeste, observa-se maior adesão aos sistemas orgânicos de produção por parte de propriedades de grande porte [5].

\section{Consumo de alimentos orgânicos}

Os meios de comunicação têm divulgado as vantagens da alimentação baseada em produtos orgânicos, o que vem contribuindo para aumentar o número de consumidores destes alimentos. Segundo Archanjo et al. [9], o crescimento do consumo não está diretamente relacionado com o valor nutricional dos alimentos, mas aos diversos significados que lhes são atribuídos pelos consumidores. Tais significados variam desde a busca por uma alimentação mais saudável, de melhor qualidade e sabor, até a preocupação ecológica de preservar o meio ambiente.

Pesquisa realizada por Archanjo et al. [9] demonstrou que os consumidores que freqüentavam a feira de produtos orgânicos de Curitiba (Paraná) apresentavam algumas peculiaridades. A maioria estabelecia uma estreita relação entre alimentação e saúde e muitos começaram a freqüentar a feira e adquirir os alimentos ali comercializados, seguindo uma prescrição médica. Alguns consumidores não demonstraram preocupação com cuidados com a saúde, adotados por meio da alimentação, e justificavam a preferência por alimentos orgânicos devido à qualidade organoléptica. À medida que despendiam, para a compra de alimentos orgânicos, mais recursos financeiros do que gastariam com a aquisição de alimentos convencionais, os consumidores acreditavam que investiam na saúde. Para os referidos consumidores, o alimento orgânico significava um meio de prevenir e até mesmo de curar doenças. Desta forma, o alimento adquire valor simbólico de medicamento, por meio do qual se busca garantir a saúde. Tais registros são comuns em outras pesquisas nacionais $[10,11]$, que abordam o consumo de alimentos orgânicos.

De acordo com Torjusen et al. [7], as pessoas que compravam alimentos orgânicos manifestaram maior preocupação no tocante às questões éticas, ambientais e de saúde. A maior parte dos consumidores estava atenta para os aspectos de produção e de processamento dos alimentos orgânicos, enfatizando os alimentos isentos de substâncias prejudiciais à saúde. Muitos dos consumidores preocupavam-se também com o conteúdo nutricional dos alimentos.

O preço dos alimentos orgânicos é considerado um fator limitante para o consumo dos mesmos, como pode ser observado por meio da totalidade das pesquisas nacionais e internacionais sobre o consumo destes alimentos $[1,10,11,12,13$, 14].

Segundo Souza \& Alcântara [15], no mercado de produtos orgânicos não existe um parâmetro definido para o estabelecimento de preços, mas sabese que as estratégias de atribuição de preços variam amplamente de acordo com o estabelecimento comercial. Por exemplo, nas grandes redes varejistas o sobre-preço cobrado em relação aos produtos convencionais é elevado, enquanto nas feiras de produtos orgânicos esta diferença é reduzida. Em média, os produtos orgânicos in natura têm um sobre-preço de $40 \%$, quando comparados aos produtos convencionais, porém, alguns produtos, 
como o trigo e o açúcar, chegam a custar (venda ao atacado), respectivamente, $200 \%$ e $170 \%$ acima do convencional.

\section{Comparações entre o valor nutritivo de alimentos orgânicos e convencionais}

Devido ao substancial aumento do interesse do consumidor pelos alimentos orgânicos, existe a necessidade de conhecer o alcance das bases científicas para as alegações de superioridade atribuídas aos produtos orgânicos.

De acordo com Bourn \& Prescott [16]; Ren $e t$ al. [17], as considerações sobre o impacto do sistema orgânico de produção na biodisponibilidade de nutrientes e o teor de compostos antioxidantes têm recebido pouca atenção, mas são importantes diretrizes para futuras pesquisas.

Estudos que compararam alimentos produzidos por meio dos sistemas orgânico e convencional foram avaliados por Bourn \& Prescott [16] sob três diferentes aspectos: valor nutricional, qualidade sensorial e segurança do alimento. Os autores afirmaram que existe reduzido número de estudos bem controlados, que sejam capazes de viabilizar uma comparação válida. Com possível exceção ao conteúdo de nitratos, os autores não verificaram fortes evidências de que alimentos orgânicos e convencionais diferissem quanto ao teor de nutrientes.

Existe uma considerável variação nos tipos e nos delineamentos de estudos que visam a identificação de diferenças entre o valor nutricional de alimentos orgânicos e convencionais. Ainda de acordo com os referidos autores [16], são quatro os tipos básicos de comparação: 1) a análise química de alimento orgânico e convencional adquiridos no comércio; 2) o efeito da fertilização na qualidade nutricional das culturas; 3) a análise dos alimentos orgânicos e convencionais provenientes de propriedades conduzidas organicamente e convencionalmente e 4) o efeito da ingestão dos alimentos orgânicos e convencionais sobre a saúde humana ou animal.

Estudos com foco no efeito do tipo de fertilizante sobre o valor nutricional do alimento e aqueles que envolvem análises de alimentos comprados no comércio não permitem a obtenção de conclusões claras sobre o impacto do sistema de produção orgânico e convencional no valor nutritivo. No primeiro tipo de comparação, embora importante, apenas um aspecto da produção é considerado, a adubação, enquanto no segundo tipo, pouco ou nada é conhecido sobre a origem dos alimentos avaliados.

Entretanto, uma vez que estudos sobre diferentes tratamentos com fertilizantes são mais baratos e mais fáceis de serem conduzidos, quando comparados aos resultados obtidos por meio de estudos envolvendo o sistema de produção como um todo, fica claro que o primeiro tipo de comparação seja o mais freqüente. Estes estudos contribuem para o conhecimento sobre os efeitos da fertilização, mas não respondem claramente às questões quanto ao efeito dos diferentes sistemas de produção sobre o valor nutricional das culturas. Informações mais precisas sobre diferenças do valor nutricional podem ser obtidas a partir da análise de alimentos provenientes de propriedades orgânicas e convencionais, pois o efeito de todo o sistema de produção sobre o valor nutricional seria realmente avaliado [16].

Toor et al. [18] verificaram a influência de diferentes tipos de fertilizantes sobre os principais componentes antioxidantes de tomates e concluíram que as fontes de adubos podem ter um expressivo efeito sobre a concentração destes compostos. A utilização de adubos orgânicos aumentou os níveis de fenólicos totais e ácido ascórbico. Porém, os autores afirmam que são necessários estudos em escala comercial, para que seja possível a confirmação de tais resultados.

Smith [19] analisou o teor de minerais de alimentos adquiridos em várias lojas da cidade de Chicago, durante o período de dois anos. As frutas (maçãs e pêras), batata e milho foram selecionados entre amostras de alimentos convencionais e orgânicos, considerando-se variedades e tamanhos similares. Os resultados revelaram que nos alimentos orgânicos, as concentrações foram superiores para os seguintes minerais: cálcio (63\%), ferro (59\%), 
magnésio (138\%), fósforo (91\%), potássio (125\%), zinco $(72,5 \%)$, sódio $(159 \%)$ e selênio $(390 \%)$. Inversamente, foi verificado menor conteúdo de alumínio (40\%), chumbo (29\%) e mercúrio (25\%). Deste modo, este estudo sugere que existem diferenças significativas, quando se estabelece a comparação entre a composição dos alimentos orgânicos e convencionais, no que diz respeito a nutrientes e contaminantes minerais.

A pesquisa de Smith [19] foi muito divulgada na mídia com alcance popular, assegurando que o alimento orgânico é mais nutritivo que o alimento convencional. Porém, o delineamento do estudo impede que os resultados sejam conclusivos, pois, aparentemente, não foi atribuída a devida atenção para verificar se os produtos rotulados como orgânicos eram de fato provenientes de um sistema orgânico de produção. Acrescenta-se, também, o fato de que não foram descritos detalhes sobre o sistema de amostragem.

Alguns pesquisadores argumentam que a melhor forma de avaliar os nutrientes é por meio da análise dos alimentos adquiridos diretamente nos locais de compra. Entretanto, esta abordagem não considera que algumas variáveis não controladas, como maturidade na colheita e tipo de cultivar e as condições de produção, possam mascarar eventuais diferenças no valor nutricional [16].

Identificar os agentes de comercialização ou produtores de alimentos orgânicos e convencionais, que atuem numa área similar, e estabelecer um protocolo experimental que permita documentar as informações do sistema de produção, tais como: a data de colheita, as condições de distribuição e transporte, as condições de armazenamento, entre outras, pode ser o mais indicado [16]. Neste caso, haveria a necessidade de estabelecer um maior número de ensaios conduzidos em diferentes áreas no sentido de poder alcançar alguma conclusão generalizada.

Pesquisa realizada por Schuphan [20], na Alemanha, durante um período de doze anos, visou a comparação entre dois padrões de aplicação de fertilizantes na produção de espinafre, batata, cenoura e repolho. Em um processo, foi utilizado um fertilizante convencional de alta solubilidade, contendo NPK (nitrogênio, fósforo e potássio), e no outro houve a adoção de adubo orgânico. Os resultados revelaram um decréscimo de $24 \%$ na produtividade, quando se utilizou adubo orgânico. No entanto, ao examinar os demais resultados, obtidos para os alimentos cultivados com a aplicação da adubação orgânica, observou-se acréscimos de matéria seca $(23 \%)$, proteína (18\%), vitamina C (28\%), açúcares totais $(19 \%)$, metionina $(23 \%)$, ferro (77\%), potássio (18\%), cálcio (10\%) e fósforo $(13 \%)$. Inversamente, verificou-se o decréscimo do sódio $(12 \%)$ e do nitrato (93\%). Embora a produção absoluta tenha sido menor com o uso dos adubos orgânicos, o substancial aumento da matéria seca, vitaminas e minerais resultou em um alimento com maior valor nutricional.

Reconhece-se que a aplicação de fertilizantes no sistema de produção afeta a composição do produto. O estudo de Schuphan [20] é freqüentemente citado com o objetivo de confirmar a superioridade do valor nutricional dos produtos orgânicos.

Premuzic et al. [21] compararam o conteúdo de ácido ascórbico de tomates cultivados com substrato orgânico aos tomates cultivados hidroponicamente e registraram um conteúdo maior de ácido ascórbico para os frutos produzidos mediante utilização de composto orgânico.

Ren et al. [17] avaliaram o conteúdo de polifenóis de cinco hortaliças (couve, repolho chinês, espinafre, alho e pimentão verde) amplamente consumidas no Japão, produzidas pelo cultivo orgânico e convencional. Os conteúdos dos orgânicos em flavonóides (quercetina) e ácido caféico foram de 1,3 a 10,4 vezes superiores aos encontrados nos convencionais, sugerindo assim a influência exercida por diferentes práticas de cultivo.

Ishida \& Chapman [22] estimaram o conteúdo total de carotenóides e, especificamente, o conteúdo de licopeno em amostras de ketchup orgânicos e convencionais. As amostras de ketchup produzidas por empresas de alimentos orgânicos apresentaram maiores teores de licopeno e de carotenóides totais. 
Caris-Veyrat et al. [23] realizaram um estudo visando à comparação do conteúdo de compostos antioxidantes presentes em tomates cultivados orgânica e convencionalmente. Os resultados, expressos em base úmida, demonstraram maior teor de vitamina $\mathrm{C}$, carotenóides e polifenóis para o tomate orgânico.

Pesquisa realizada no Brasil por Borguini [24] registrou que tomates provenientes de sistema orgânico de produção apresentaram maior teor de fenólicos totais e de ácido ascórbico do que o tomate produzido por cultivo convencional.

Alguns pesquisadores mantêm-se atentos para controlar variáveis como localização da propriedade, cultivar e maturação na colheita, como uma maneira de reduzir o número de fatores potenciais que podem afetar o valor nutricional. Os estudos comparando o sistema de produção como um todo têm relativa vantagem, pois evitam atribuir importância a fatores individuais no valor nutricional dos alimentos. Além disso, é importante que as propriedades venham sendo manejadas orgânica ou convencionalmente por um considerável período de tempo.

Segundo Bourn \& Prescott [16], a ampla gama de fatores que pode afetar a composição dos alimentos (genéticos, práticas agronômicas, clima e condições de pós-colheita) faz com que as pesquisas sobre o valor nutricional de alimentos, produzidos orgânica e convencionalmente, tornem-se difíceis de serem estabelecidas e seus resultados interpretados de forma consistente. No entanto, devido ao crescente interesse pelo tema e ao aumento da produção e do consumo de alimentos orgânicos, maior número de pesquisas deve ser implementado neste sentido.

Existe uma tendência, que pode ser observada por meio dos resultados das pesquisas anteriormente citadas, que indica maior conteúdo de nutrientes para os alimentos produzidos organicamente.

\section{Resíduos de pesticidas em alimentos}

O maciço uso de produtos químicos na agricultura teve início na década de 50, logo após o final da Segunda Guerra Mundial. De acordo com os alvos contra os quais são destinados, os citados produtos químicos são denominados inseticidas, fungicidas, herbicidas, nematicidas, entre outros [25]. Este conjunto de produtos químicos recebeu as seguintes denominações: defensivos agrícolas, pesticidas, praguicidas, produtos fitossanitários ou agrotóxicos (este último termo restrito ao Brasil, por força da Lei no 7.802/89).

O aumento do uso de produtos químicos na agricultura tem gerado preocupação crescente, quanto aos riscos à saúde humana e ao meio ambiente. Esta preocupação decorre de casos de doenças registradas em seres humanos e das alterações ambientais, que parecem ter como agentes etiológicos os agrotóxicos. De acordo com Kotaka \& Zambrone [25], no Brasil, o uso de produtos químicos na agricultura depende do registro concedido pelo Ministério da Agricultura, Pecuária e Abastecimento (MAPA), condicionado à autorização do Ministério da Saúde (MS) e do Instituto Brasileiro do Meio Ambiente e Recursos Naturais Renováveis (IBAMA).

Uma das maiores preocupações dos consumidores com relação ao uso de agrotóxicos na agricultura é o conhecimento do grau de contaminação, a ponto de saber se os alimentos estão contaminados com resíduos tóxicos, que possam comprometer a saúde. Conforme a Portaria no 03, de 16 de janeiro de 1992, da Agência Nacional de Vigilância Sanitária (ANVISA) [26], resíduo de agrotóxico consiste em "substância ou mistura de substâncias remanescente ou existente em alimentos ou no meio ambiente, decorrente do uso ou da presença de agrotóxicos e afins, inclusive quaisquer derivados específicos, tais como: produtos de conversão e de degradação, metabólitos, produtos de reação e impurezas, consideradas tóxicas e ambientalmente importantes".

A agricultura orgânica baseia-se no emprego mínimo de insumos externos. Devido à contaminação ambiental, as práticas de agricultura orgânica não podem garantir a ausência total de resíduos. No entanto, é possível adotar métodos destinados a reduzir ao mínimo a contaminação do ar, do solo e da água. Os manipuladores, processadores e vendedores envolvidos no manuseio 
dos produtos orgânicos aderem às normas que mantém a integridade dos produtos da agricultura orgânica [4].

A produção orgânica de alimentos surge como uma alternativa ao quadro de contaminação química dos alimentos, buscando oferecer produtos isentos de resíduos químicos. Os atributos de qualidade dos produtos obtidos por meio da agricultura orgânica, como a ausência de resíduos químicos ou aditivos sintéticos, representam elevado grau de afinidade com o conceito de segurança do alimento, que inclui a aquisição pelo consumidor de alimentos de boa qualidade, livre de contaminantes de natureza química (pesticidas, aditivos), física ou biológica [15].

Os consumidores freqüentemente citam a preocupação com a saúde como a principal motivação para consumir alimentos orgânicos. A possível ausência de agrotóxicos é apontada como o principal atributo destes alimentos. Considerandose a proibição da aplicação de pesticidas químicos sintéticos no sistema orgânico de produção, seria razoável assumir que alimentos produzidos organicamente, em geral, contenham menores níveis de resíduos de pesticidas do que aqueles produzidos convencionalmente [16].

A quantidade de pesticidas utilizada na produção de um vegetal varia amplamente de cultura para cultura. Um considerável número de fatores irá afetar a presença de resíduos em alimentos, incluindo o estágio de desenvolvimento da cultura em que o pesticida foi aplicado, a persistência do produto, o uso de pesticida no pós-colheita e o nível de pesticidas presentes no meio ambiente [16]. Devido à tendência de uso de pesticidas na agricultura convencional, é provável que os consumidores de orgânicos estejam, no mínimo, consumindo menores quantidade e tipos de resíduos. Porém, ainda não foi elucidado se tais resultados envolvem benefícios à saúde dos consumidores.

Ainda de acordo com Bourn \& Prescott [16], considerando-se que muitos consumidores optam por comprar alimentos produzidos organicamente por acreditarem que estes alimentos possuem um menor nível de resíduos de pesticidas, seria interessante implementar pesquisas com vistas à confirmação de tal hipótese. No futuro, com a tendência de declínio do uso de pesticidas nas culturas produzidas convencionalmente, devido às técnicas de produção, como o Manejo Integrado de Pragas, pode ser que a questão dos resíduos de pesticidas venha a se revelar menos importante para a decisão dos consumidores pela compra de alimentos orgânicos em comparação às outras questões.

Conforme a Portaria no 03, de 16 de janeiro de 1992, da Agência Nacional de Vigilância Sanitária (ANVISA), Ingestão Diária Aceitável (IDA) é a "quantidade máxima que, ingerida diariamente durante toda a vida, parece não oferecer risco apreciável à saúde, à luz dos conhecimentos atuais. É expressa em miligramas (mg) do agrotóxico por quilograma ( $\mathrm{kg}$ ) de peso corpóreo (mg / kg p.c.)". De acordo com a mesma Portaria, Limite Máximo de Resíduo (LMR) significa a "quantidade máxima de resíduo de agrotóxico legalmente aceita no alimento, em decorrência da aplicação adequada numa fase específica, desde sua produção até o consumo, expressa em partes (em peso) do agrotóxico ou seus derivados por um milhão de partes de alimento (em peso) (ppm ou $\mathrm{mg} / \mathrm{kg}$ )" [26].

Para garantir a qualidade dos alimentos e a segurança para a população, o Ministério da Saúde exige que sejam realizadas análises de resíduos de agrotóxicos comprobatórias da segurança do alimento em todas as culturas para as quais ele será registrado. Os níveis de resíduos, eventualmente detectados nos alimentos, devem ser inferiores ao LMR estabelecido após a realização de todos os estudos toxicológicos necessários para efeito de registro. Os testes toxicológicos realizados têm como um de seus principais objetivos determinar qual a quantidade que poderá ser ingerida pelas pessoas (IDA), sem que isto provoque qualquer tipo de dano à sua saúde [27].

Em muitos países, a presença e a quantidade de resíduos de agrotóxicos em alimentos nacionais e importados são monitoradas para assegurar que a população tenha acesso a uma dieta que não ultrapasse o nível de tolerância (LMR) recomendado, com base nos estudos de Ingestão Diária Aceitável. 
Para tanto, os Limites Máximos de Resíduos são estabelecidos internacionalmente e divulgados pela Food and Agriculture Organization (FAO). Este monitoramento pode contribuir para ampliar a confiança do consumidor na qualidade dos alimentos ofertados e minimizar possíveis riscos à saúde pública[27].

A legislação brasileira previu normas que visam a regulamentação do uso de defensivos na agricultura, mas, salvo em alguns estados que dispõem de fiscalização efetiva, a obediência às leis ainda esbarra em questões sócio-culturais peculiares de cada região [28].

A presença de resíduos de defensivos em alimentos, somada à contaminação da água, constituem risco para a população em geral e representam, sem dúvida, um grande problema de saúde pública no Brasil. Com o objetivo de ampliar o nível de conhecimento da situação, Araújo et al.[28] analisaram a utilização de defensivos em tomates produzidos no estado de Pernambuco. Outras motivações para a pesquisa referem-se ao fato da grande importância socioeconômica da cultura deste fruto e o potencial risco epidemiológico relacionado à saúde dos consumidores dos referidos alimentos. No modelo dominante de produção, a cultura do tomate demanda uso intensivo de agroquímicos e este fruto integra o hábito alimentar da população em geral.

De acordo com os referidos autores [28], todas as áreas integrantes da pesquisa careciam de ações que visassem à proteção da saúde dos trabalhadores rurais, que lidavam com os defensivos e, também, de medidas de proteção ao meio ambiente, que se revelou gravemente comprometido. Os autores relataram ainda que produtos sem registro autorizado para o uso na produção de tomates eram comumente utilizados pelos agricultores. Tal situação era agravada pelo fato de não existir qualquer tipo de controle sistemático da presença de resíduos de defensivos nos alimentos ou dos produtos comercializados no estado de Pernambuco. Observou-se ainda a escassez de campanhas efetivas com vistas à orientação, apoio e educação dos produtores envolvidos. Foi registrado o desconhecimento, por parte dos produtores e aplicadores de defensivos, no que se refere aos efeitos tóxicos para a saúde e o meio ambiente, associados ao uso indevido dos produtos não-autorizados.

Caldas \& Souza [29], a partir de dados de consumo de alimentos registrados pela Pesquisa de Orçamentos Familiares, realizada pelo IBGE, em 1995-96, identificaram os alimentos que mais contribuíram para a Ingestão Diária Máxima Teórica (IDMT) de pesticidas. O cereal (arroz) e a leguminosa (feijão), de expressivo consumo pela população brasileira, as frutas, principalmente as cítricas, e o tomate foram alimentos que exerceram papel preponderante para a elevação da ingestão.

O estudo de Lourenço [27] teve por objetivo discutir as possíveis interações entre os agrotóxicos, de uso autorizado em produtos de origem vegetal, como tomate, banana e maçã, consumidos nas principais regiões metropolitanas brasileiras, e o possível risco à saúde humana. $\mathrm{O}$ autor concluiu que existe perigo à saúde humana, decorrente de tais interações, com efeitos danosos e de diferentes proporções. À medida que outros estudos forem realizados nesta linha de pesquisa, os resultados gerados permitirão que as autoridades competentes harmonizem os interesses agrícolas com a proteção da saúde pública. Neste contexto, a população tem o direito de conhecer os riscos a que está exposta ao ingerir cada alimento e, a partir disso, optar pelo que prefere consumir.

Ainda de acordo com Lourenço [27], os danos à saúde humana, devido à ingestão de resíduos de agrotóxicos em alimentos, só poderão ser minimizados pelo uso restrito, controlado e racional destes produtos na agricultura. Existe uma necessidade urgente de ações na área de saúde pública, para que seja possível uma identificação rápida e segura das intoxicações causadas por agrotóxicos.

Também de acordo com Lourenço [27], como medida de segurança, a população tenta evitar os possíveis riscos da presença de resíduos de agrotóxicos em alimentos, entre outras práticas, comprando alimentos orgânicos que, em geral, são mais caros que os convencionais e preferindo a aquisição de produtos de origem vegetal com selo de qualidade e consumindo alimentos da época. 
Vale registrar que o consumo de alimentos seguros significa a promoção da saúde e manutenção da qualidade de vida da população. A garantia de alimento relativamente livre de contaminantes é essencial para a prevenção de doenças, principalmente em um país como o Brasil, onde uma parte considerável da população enfrenta sérios problemas relativos aos distúrbios nutricionais e limitações no tocante ao acesso ao sistema público de saúde [29].

Considerando-se a permissão do uso de pesticidas na agricultura convencional, espera-se encontrar menores níveis de resíduos nos produtos orgânicos. Porém, existem poucos registros sobre os níveis de pesticidas em alimentos orgânicos [30].

Segundo Pussemier et al. [31], quando são comparados os efeitos do sistema de produção na segurança do alimento, há evidências de que, com relação à presença de resíduos de pesticidas, os alimentos orgânicos apresentam uma clara vantagem sobre os convencionais.

Kouba [32] registrou que os alimentos orgânicos de origem animal apresentavam menores níveis de pesticidas e de drogas de uso veterinário.

Moore et al. [33] analisaram alimentos infantis de diversas marcas, elaborados com produtos provenientes do cultivo orgânico e convencional, quanto à presença de resíduos de pesticidas, e não encontraram tais resíduos em nenhum dos produtos analisados. Portanto, neste caso, não houve diferença entre orgânicos e convencionais.

Baker et al. [34] avaliaram dados relativos à presença de resíduos de pesticidas em alimentos produzidos por meio do cultivo convencional, do Manejo Integrado de Pragas e do sistema orgânico. Segundo os autores, os alimentos orgânicos continham menor número de resíduos de pesticidas, quando comparados àqueles provenientes dos outros sistemas de produção, e, quando presentes, tais resíduos estavam em menor quantidade nos alimentos orgânicos.

Rekha et al. [35] analisaram amostras de trigo e arroz produzidas em sistema orgânico e convencional, quanto à presença de resíduos de pesticidas. Considerando que não foi encontrada nem mesmo quantidade-traço de resíduos nos alimentos produzidos organicamente, os autores recomendaram o consumo de arroz e trigo orgânicos.

Em pesquisa realizada no Brasil, Borguini [24] registrou que a forma de cultivo foi um fator determinante em relação à presença de resíduos de pesticidas e, como esperado, de acordo com as diretrizes para produção orgânica de alimentos [36], o tomate orgânico não apresentou tais resíduos.

Portanto, de acordo com os resultados das pesquisas anteriormente citadas, os alimentos genuinamente orgânicos contêm menores níveis ou, simplesmente, não apresentam resíduos de pesticidas, quando comparados aos alimentos convencionais. Os dados sugerem que os consumidores, que buscam reduzir sua exposição aos resíduos de pesticidas, podem optar pela aquisição de alimentos orgânicos.

\section{Considerações Finais}

As informações indicam que existem diferenças relativas à qualidade nutritiva, quando se estabelece uma comparação entre os alimentos produzidos pelos métodos orgânico e convencional. Entretanto, as evidências não são suficientes para assumir, de forma definitiva, a superioridade do alimento produzido organicamente, quanto à qualidade nutritiva e aos benefícios do seu consumo para a saúde do consumidor. Deste modo, recomenda-se que pesquisas sejam desenvolvidas, controlando-se a ampla gama de fatores que podem afetar a composição dos alimentos, tais como: fatores genéticos, práticas agronômicas, clima e condições de pós-colheita, entre outros.

Os alimentos orgânicos possuem menores níveis de resíduos de pesticidas ou, simplesmente, não contêm quantidades detectáveis de tais resíduos. Porém, a escassez de dados sobre a presença de resíduos de pesticidas em alimentos produzidos organicamente não permite conclusões definitivas para estabelecer alguma diferença entre alimentos orgânicos e convencionais. 


\section{Referências Bibliográficas}

1. Borguini RG \& Mattos FL. Análise do Consumo de Alimentos Orgânicos no Brasil. In: Anais do XL Congresso Brasileiro de Economia e Sociologia Rural, 2002; 28 - 31 julho; Passo Fundo; Brasília: SOBER, 2002. p.38.

2. Penteado SR Introdução à agricultura orgânica. Campinas: Grafimagem; 2000.

3. Paschoal AD Produção orgânica de alimentos: agricultura sustentável para os séculos XX e XXI. Piracicaba: EDUSP, 1994.

4. FAO/Organização Mundial da Saúde. Codex Alimentarius: Alimentos Producidos Orgánicamente, 2001 [citado em 2001 set 05]. Disponível em: http:/ /www.fao.org/DOCREP/005/Y2772S/ Y2772S00.HTM

5. Souza MCM. Aspectos institucionais do sistema agroindustrial de produtos orgânicos. Informações Econômicas. 2003; 33(3): 7-16.

6. Brasil. Ministério da Agricultura, Pecuária e do Abastecimento. Lei Federal no 10.831 de dezembro de 2003. Dispõe sobre normas para a produção de produtos orgânicos vegetais e animais. Diário Oficial da República Federativa do Brasil, Brasília, 23 dez 2003. Seção 1, p.11.

7. Torjusen H, Lieblein G, Wandel M, Francis CA. Food system orientation and quality perception among consumers and producers of organic food in Hedmark County, Norway. Food Qual Preferences. 2001; 12: 207-216.

8. Ormond JGP, Paula SRL, Faveret Filho P, Rocha LTM. Agricultura orgânica: quando o passado é futuro. BNDS Setorial 2002, 15: 3-34.

9. Archanjo LR, Brito KFW, Sauerbeck S. Os alimentos orgânicos em Curitiba: consumo e significado. Cadernos de Debate. 2001; 8: 1-6.

10. Assis RL, Arezzo DC, De-Polli H. Consumo de produtos da agricultura orgânica no estado do Rio de Janeiro. Rev Administração. 1995; 30(1): 84-89.
11. Cerveira R, Castro MC. Consumidores de produtos orgânicos da cidade de São Paulo: características de um padrão de consumo. Informações Econômicas. 1999; 29(12): 7-19.

12. Glaser LK, Thompson GD. Demand for organic and conventional frozen vegetables. In: American Agricultural Economics Association Annual Meeting, Nashville, 1999. Selected Paper. Ames: American Agricultural Economics Association, 1999 [citado em 2002 may 15]; p.1-14. Disponível em: http:// www.ers.usda.gov/publications/aib777/ aib777rf.pdf

13. Lohr L. Factors affecting international demand and trade in organic food products. Athens: University of Georgia, Department of Agricultural and Applied Economics, 2000 [citado em 2002 may 15]; p.1-35. (Faculty Series 00-20) Disponível em: http:/ /www.ers.usda.gov/publications/wrs011/ wrs011j.pdf

14. Wier M, Andersen LM. Studies on consumer demand for organic foods: a survey. Copenhagen: AKF, 2001 [citado em 2002 may 15]; 15p. (Project on consumer demand for organic foods: domestic and foreign market perspectives. Working Paper, 1) Disponível em: http://www.akf.dk/organicfoods/ Papers/wp1-mw.pdf

15. Souza APO, Alcântara RLC Alimentos orgânicos: estratégias para o desenvolvimento do mercado. In: Neves MF, Castro LT (org) Marketing e estratégia em agronegócios e alimentos. São Paulo: Atlas; 2003.

16. Bourn D, Prescott J. A Comparison of the nutritional value, sensory qualities and food safety of organically and conventionally produced foods. Crit Rev Food Sci Nutr. 2002; 42(1): 1-34.

17. Ren H, Endo H, Hayashi T. Antioxidative and antimutagenic activities and polyphenol content of pesticide-free and organically cultivated green vegetables using water-solubre chitosan as a soil modifier and leaf surface spray. J Sci Food Agric. 2001; 81: 1426-1432.

18. Toor RK, Savage GP, Heeb A. Influence of 
different types of fertilizers on the major antioxidant components of tomatoes. J Food Comp Anal. 2006; 19(1): 20-27.

19. Smith BL. Organic foods vs. supermarket foods: element levels. J Appl Nutr. 1993; 45(1): 35-39.

20. Schuphan W. Nutritive value of crops as influenced by organic and inorganic fertilizer treatment. Qualitas Plantarum: plant foods for human nutrition. 1974; 23(4): 333-358.

21. Premuzic Z, Bargiela M, Garcia A, Rondina A, Lorio A. Calcium, iron, potassium, phosphorus and vitamin $\mathrm{C}$ content of organic and hidroponic tomatoes. Hortscience. 1998; 33: 255-257.

22. Ishida BK, Chapman MH. A comparison of carotenoid content and total antioxidant activity in catsup from several commercial sources in the United States. J Agri Food Chem. 2004; 52: 8017-8020.

23. Caris-Veyrat C, Amiot MJ, Tyssandier V, Grasselly D, Buret M, Mikoljozak M, Guilland JC, Bouteloup-Demange C, Borel P. Influence of organic versus conventional agricultural practice on the antioxidant microconstituent content of tomatoes and derived purees; consequences on antioxidant plasma status in humans. J Agric Food Chem. 2004; 52: 6503-6509.

24. Borguini RG. Avaliação do potencial antioxidante e de algumas características físico-químicas do tomate (Lycopersicon esculentum) orgânico em comparação ao convencional [tese]. São Paulo: Universidade de São Paulo; 2006. 161p.

25. Kotaka ET, Zambrone FA. Contribuições para a construção de diretrizes de avaliação do risco toxicológico de agrotóxicos. Campinas: ILSI Brasil; 2001.

26. ANVISA. Portaria $n^{\circ}$ 3, de 16.1.1992: ratifica os termos das "diretrizes e orientações referentes à autorização de registros, renovação de registro e extensão de uso de agrotóxicos e afins - $\mathrm{n}^{\circ} 1$, de 09.12.1991", publicadas no D.O.U. em 13.12.1991. Diário Oficial da União, Brasil, 4 fev. 1992.
27. Lourenço RC. Discussão sobre o risco das interações de agrotóxicos na dieta brasileira [dissertação]. São Paulo: Universidade de São Paulo; 2003.

28. Araújo ACP, Nogueira DP, Augusto LGS. Impacto dos praguicidas na saúde: estudo da cultura de tomate. Rev de Saúde Pública. 2000; 34(3): 309313.

29. Caldas ED, Souza LCK. Avaliação de risco crônico da ingestão de resíduos de pesticidas na dieta brasileira. Rev Saúde Pública. 2000; 34(5): 529-537.

30. Siderer Y, Maquet A, Anklam E. Need for research to support consumer confidence in the growing organic food market. Trends Food Sci Technol. 2005; 16(8): 332-343.

31. Pussemier L, Larondelle Y, Peteghem CV, Huyghebaert A. Chemical safety of conventionally and organically produced foodstuffs: a tentative comparison under Belgian conditions. Food Control. 2006; 17: 14-21.

32. Kouba M. Quality of organic animal products. Livestock Production Sci. 2003; 80: 33-40.

33. Moore VK, Zabik ME, Zabik MJ. Evaluation of conventional and "organic" baby food brands for eight organochlorine and five botanical pesticides. Food Chem. 2000; 71: 443-447.

34. Baker BP, Benbrook CM, Groth III E, Benbrook KL. Pesticide residues in conventional, integrated pest management (IPM)-grown and organic foods: insights from three US data sets. Food Additives and Contaminants. 2002; 19(5): 427-446.

35. Rekha, Naik SN, Prassad R. Pesticide residue in organic and conventional food-risk analysis. J Chem Health Safety. 2006; 13(6): 12-19.

36. Brasil. Ministério da Agricultura, Pecuária e do Abastecimento. Lei Federal no 10.831 de dezembro de 2003. Dispõe sobre normas para a produção de produtos orgânicos vegetais e animais. Diário Oficial da República Federativa do Brasil, Brasília, 23 dez 2003. Seção 1, p.11. 


\section{Autores}

Renata Galhardo Borguini - Agência Paulista de Tecnologia dos Agronegócios. Secretaria de Agricultura e Abastecimento do Estado de São Paulo. Correspondência: Av. Prof. Manoel César Ribeiro, 1920
Caixa Postal 07, CEP: 12400-970 Pindamonhangaba/SP Fax: (12) 3642-1812. E-mail: renata@aptaregional.sp.gov.br

Elizabeth A. Ferraz da Silva Torres - Departamento de Nutrição. Faculdade de Saúde Pública. Universidade de São Paulo 